\title{
The Electromagnetic Spectrum: Current and Future Applications in Oncology
}

\author{
Allison RR \\ $21^{\text {st }}$ Century Oncology, 801 WH Smith Boulevard, Greenville, NC, USA
}

Future Oncol. 2013;9:657-667 doi: 10.2217/fon.13.12

\section{EDITORIAL COMMENT}

The electromagnetic spectrum is the foundation of physics. The electromagnetic spectrum includes radio waves, infrared radiation, visible light and ultraviolet radiation, $\mathrm{X}$-rays, and gamma-rays. The electromagnetic spectrum is composed of waves of various energies that interact with matter. These energy sources can be used for cancer ablation. Different types of energy in the electromagnetic spectrum can be a potential application for the diagnosis and treatment of cancer. Radio waves, microwaves and infrared waves, electroporation, light-based therapies [e.g. photo-chemotherapy, photodynamic therapy (PDT)], and radiation-based therapies are new opportunities for cancer treatment. Radio frequency ablation (RFA), microwaves, ultrasounds, lasers and magnetic resonance imaging are actual members of this concept. Many of them have some disadvantages such as tissue warming, lack of miniaturized probes and high cost. In the context of warming, new dosimetry apparatus are being developed for cancer ablation. It is also likely that for PDT, perhaps RFA and MW therapy will be pioneer of nanoparticle-based treatment. Ultrasound and laser energy could also heat nanoparticles for precise therapy. These data would usher in a new age of minimally-invasive oncologic therapy. On the other hand, radiation therapy is a well known technique for cancer treatment. It is anticipated that image-guided radiation delivery systems will enable a greater degree of personalization of radiotherapy thus increase the precision and accuracy of radiation delivery. Robotic radiosurgery devices are becoming far more common. No doubt nanoparticles directed to the tumor will allow for enhanced lesion imaging to better target the tumor. The future will also bring more widespread use of particle (Hadron) therapy as particle radiations (i.e. protons and carbon ions). Clearly, the future application of the electromagnetic spectrum will help ultra-precise treatment of cancer. Moreover, I believe that more accurate and timely diagnosis of cancer may be possible with the discovery of all the parts of the electromagnetic spectrum.

Fehmi Narter. Prof, MD PhD

๑Copyright 2019 by the Association of Urological Surgery / Journal of Urological Surgery published by Galenos Publishing House. 